\title{
Cutaneous Manifestations in Four Patients with COVID-19
}

\author{
Ana Gorgulho, Maria Margarida Andrade, Ana Paula Antunes, Inês Nabais, Filipa Taborda, Marta Dias Ramos, Ana Maria Grilo \\ Hospital de Cascais, Cascais, Portugal
}

Doi: 10.12890/2020_002029 - European Journal of Case Reports in Internal Medicine - ๑ EFIM 2020

Received: $26 / 09 / 2020$

Accepted: 01/10/2020

Published: 05/11/2020

How to cite this article: Gorgulho A, Andrade MM, Antunes AP, Nabais I, Taborda F, Dias Ramos M, Grilo AM. Cutaneous manifestations in four patients with COVID-19. EJCRIM 2020;7: doi:10.12890/2020_002029.

Conflicts of Interests: The Authors declare that there are no competing interests.

This article is licensed under a Commons Attribution Non-Commercial 4.0 License

\section{ABSTRACT}

As the numbers of SARS-CoV-2 infections increased globally, reports of cutaneous manifestations started to emerge. We describe several patients with COVID-19 who presented with skin changes. We noted such manifestations in four out of 110 patients (3.63\%) and describe the clinical situation of each of these patients. Each patient had either a maculopapular or a urticariform rash. These manifestations have a broad differential diagnosis and it was difficult to exclude drug reactions. We hope to raise awareness of this possible manifestation of COVID-19 in order to raise suspicion of this diagnosis among clinicians when they encounter patients with fever and rash. Larger series that also include patients with mild disease and skin biopsies may be useful.

\section{LEARNING POINTS}

- Cutaneous manifestations can occur as part of COVID-19, so clinicians should be suspicious of this diagnosis in patients with fever and rash.

- The differential diagnosis is large and drug reactions are difficult to rule out.

\section{KEYWORDS}

COVID-19, SARS-CoV-2, dermatology, skin, rash

\section{CASE DESCRIPTIONS}

Our regional hospital in Cascais, Portugal, set up a dedicated ward for patients with SARS-CoV-2 infection. From the end of March until the beginning of June, 110 COVID-19 patients were admitted to this ward. Dermatological manifestations were seen in four patients (3.63\%). Here we describe the clinical presentations of these patients.

\section{Case 1}

We present the case of a 35-year old woman who was obese and a smoker. She had a cough for 5 days and a fever for 2 days before she was diagnosed with COVID-19 with bilateral pneumonia. She was started on amoxicillin plus clavulanate, hydroxychloroquine, azithromycin and oseltamivir (as this was standard treatment at that time in our hospital). She remained febrile for 10 days. On the last day of fever and 2 days after stopping all antimicrobials, she developed a pruriginous maculopapular rash on her chest, back, buttocks and arms (Fig. 1). On the day after the rash appeared, she also developed pleuritic chest pain and a pulmonary thromboembolism was diagnosed. She was started on an anti-histamine and hypocoagulation with enoxaparin $1 \mathrm{mg} / \mathrm{kg} / \mathrm{day}$. The rash lasted for 8 days. 


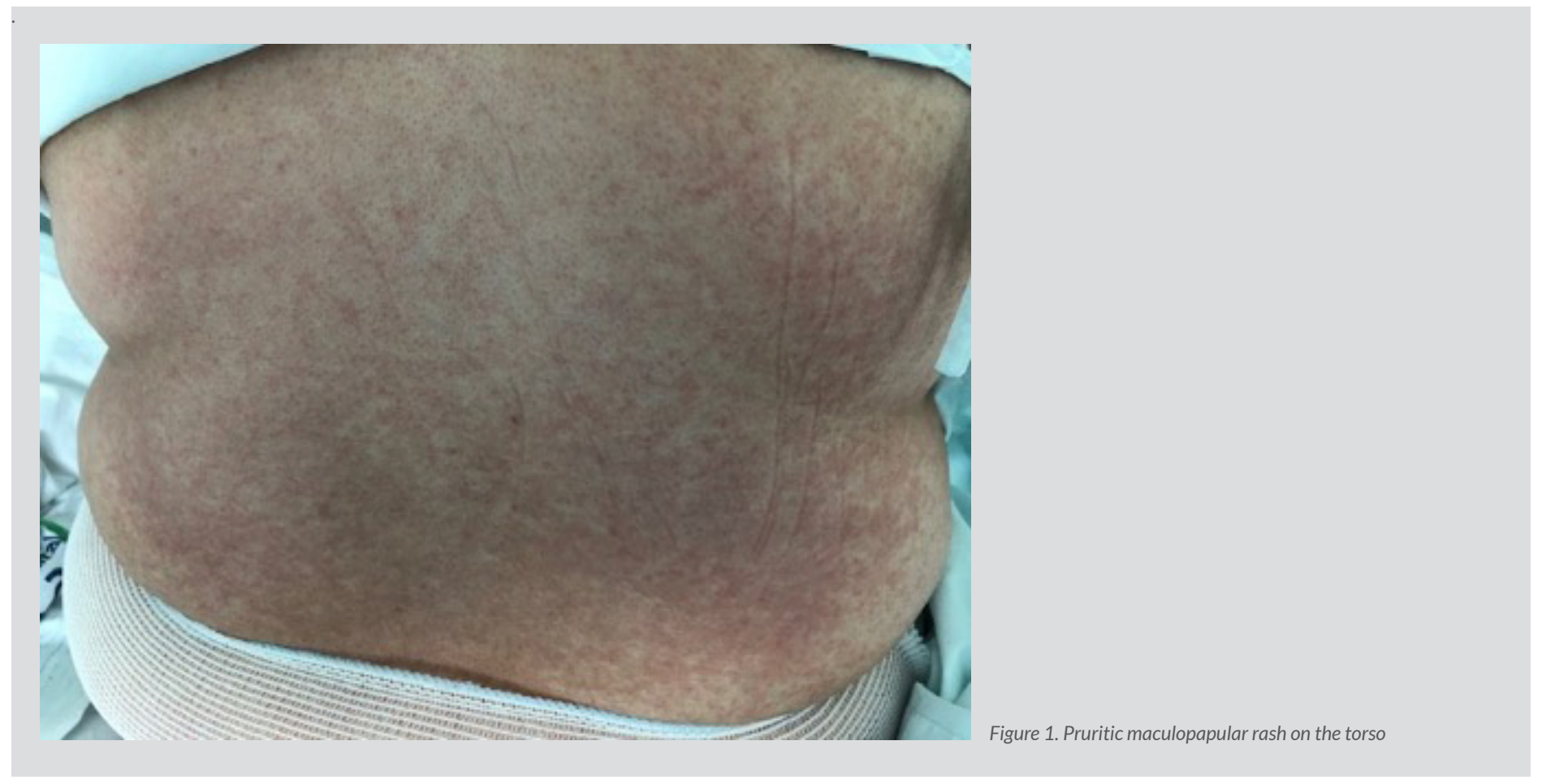

\section{Case 2}

We present the case of an 83-year old man with a prosthetic mitral valve who was admitted due to fever. He had Staphylococcus epidermidis bacteraemia and had been on vancomycin, gentamicin and rifampicin for 8 days when he developed a cough and fever recurrence. A first test for SARS-CoV-2 on that day was negative. As the patient was not improving and endocarditis was excluded, the antimicrobials were stopped after 14 days. On the same day, the patient developed a non-pruritic morbilliform rash with centripetal distribution (mainly on the trunk and thighs) (Fig. 2), which resolved after 6 days. In addition to the antimicrobials, the only recently prescribed new drug was paracetamol. A new test was performed and the patient was diagnosed with COVID-19. There were no signs of viral pneumonia on chest CT. After the cough and rash resolved, the patient remained febrile and had intense lower back pain. Spondylodiscitis was diagnosed.

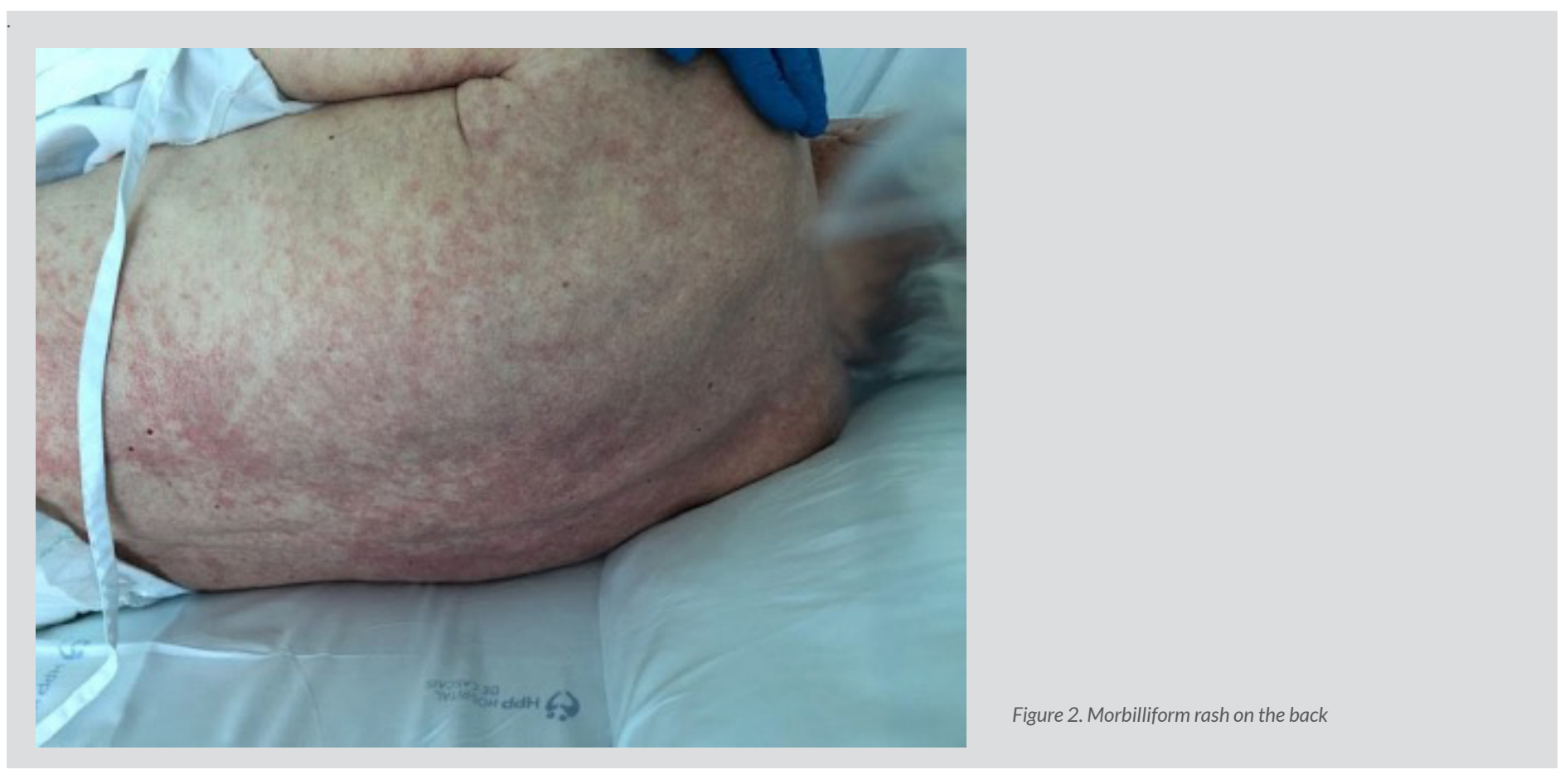


Case 3

We present the case of an 81-year old woman with known hypertensive heart disease with heart failure and atrial fibrillation, who was admitted to our COVID-19 ward. The diagnosis of SARS-CoV-2 infection was made while she was asymptomatic, as a number of residents in her nursing home had been diagnosed with COVID-19. The patient eventually developed fever, dry cough and dyspnoea and was admitted to the hospital with bilateral pneumonia. She completed 7 days of ceftriaxone and 5 days of azithromycin and oseltamivir. On the day after all antimicrobials had been stopped, the patient remained febrile and a pruritic maculopapular rash appeared on her limbs, with some urticariform areas (Figs. 3 and 4). This patient was taking multiple medications but none had been recently introduced. The rash disappeared after 6 days, simultaneously with fever remission.

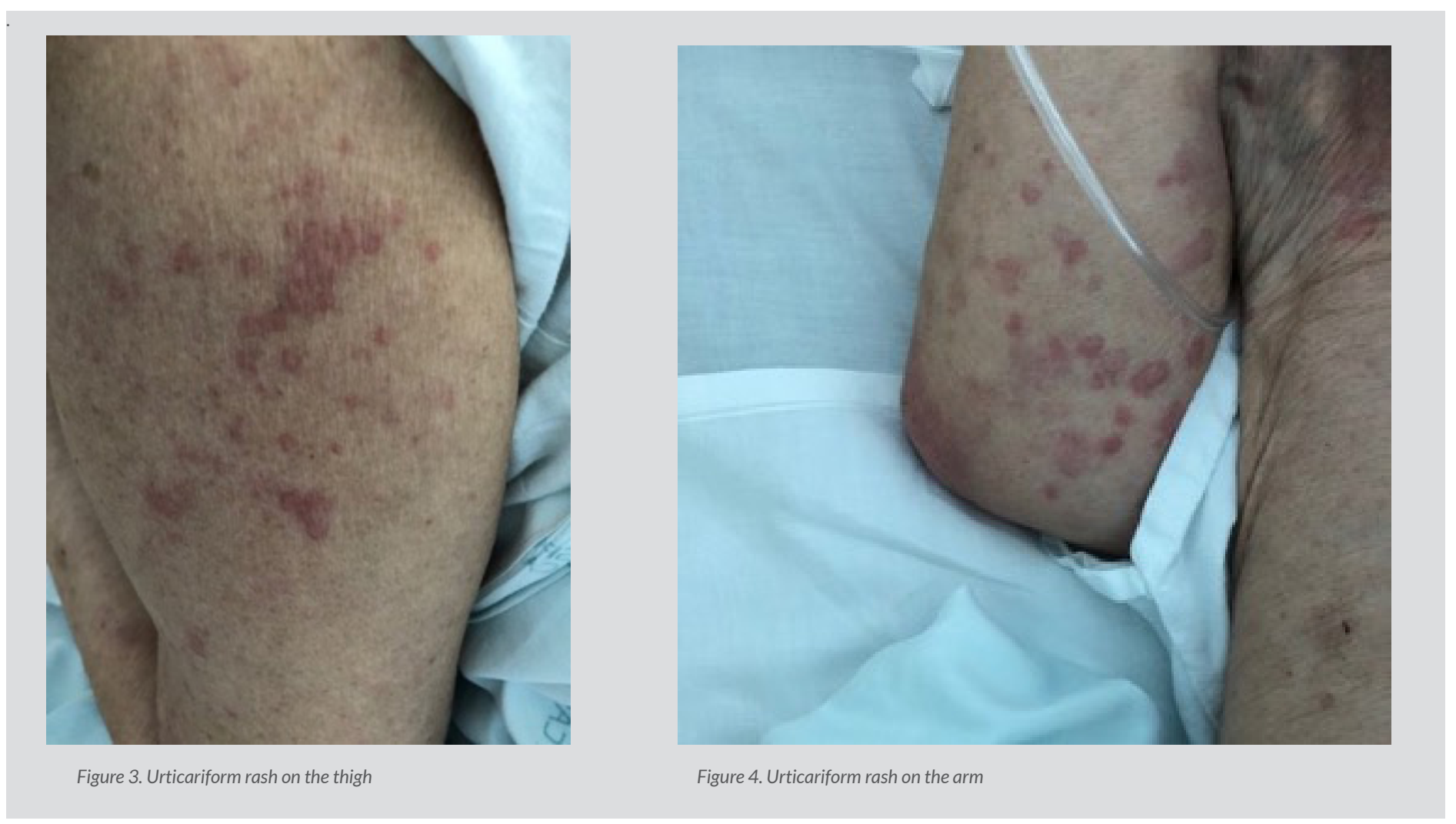

Case 4

We report the case of a 63-year old woman who was diagnosed with COVID-19 and, 10 days after symptom began, was admitted to hospital and diagnosed with bilateral viral pneumonia. She had a fever for a total of 12 days. Two days after fever resolution, she developed a pruritic, erythematous papular rash on her torso (Fig. 5). When the rash developed she had been on ceftriaxone and azithromycin for 5 days. Antihistamine drugs were started with pruritus improvement; the rash resolved after 10 days.

\section{METHODS AND PROCEDURES}

Each case was observed in consultation with a dermatologist. Drug reactions were hard to completely rule out as all of these patients were on at least one antimicrobial before the rash appeared.

\section{DISCUSSION}

As the numbers of SARS-CoV-2 cases increased globally, more reports of cutaneous manifestations were published. Galván Casas et al. reviewed the literature on Spanish patients and classified dermatological patterns in COVID-19 patients into acral areas of erythema with vesicles or pustules (pseudo-chilblain), other vesicular eruptions, urticarial lesions, maculopapular eruptions and livedo or necrosis ${ }^{[1]}$. The frequency of these manifestations has not yet been determined, but Italian colleagues report that as many as $20 \%$ of COVID-19 patients develop cutaneous manifestations, while others describe these in only $1 \%$ of patients ${ }^{[2,3]}$. However, as most reports mainly include patients admitted to hospital, mild cutaneous forms are most likely under-represented. 


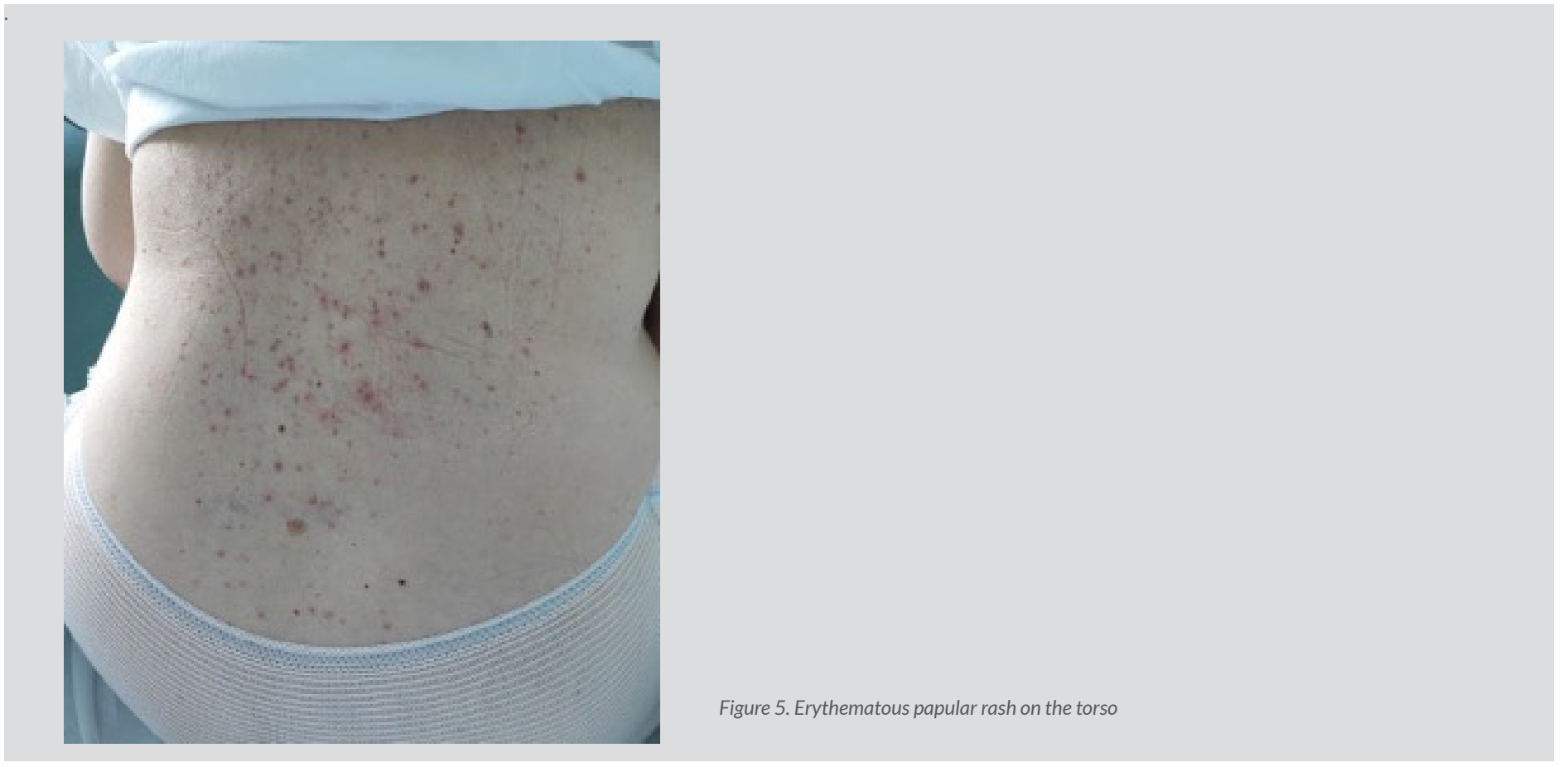

None of our patients had severe disease or needed mechanical ventilation, which agrees with the low percentage of patients with urticarial or maculopapular rash who required ICU admission or mechanical ventilation in the Galván Casas et al. report.

Regarding the temporal relationship between cutaneous manifestations and other symptoms, each patient we describe developed cutaneous involvement at the same time as or after developing other symptoms. This also agrees with the findings of Galván Casas et al. who report that urticarial and maculopapular rashes mainly appear at the same time as or after other symptoms ${ }^{[1]}$.

We acknowledge that maculopapular and urticarial lesions are common and can have a number of different causes. However, we believe that it is important to highlight them and that one of the strengths of our report is that we have included photographs.

More uncommon manifestations such as pseudo-chilblain or vesicular lesions might be more helpful in raising suspicion of COVID-19 but, as we are still experiencing active community transmission, a patient with maculopapular or urticarial lesions and fever should also raise the possibility of this diagnosis. We hope that we have encouraged other colleagues to keep an eye out for these manifestations. Large-scale studies with biopsies that can help elucidate potential mechanisms are needed.

\section{REFERENCES}

1. Galván Casas C, Catala A, Carretero Hernandez G, Rodriguez-Jimenez P, Fernandez-Nieto D, Rodriguez-Villa Lario A, et al. Classification of the cutaneous manifestations of COVID-19: a rapid prospective nationwide consensus study in Spain with 375 cases. Br J Dermatol 2020;183(1):71-77.

2. Recalcati S. Cutaneous manifestations in COVID-19: a first perspective. J Eur Acad Dermatol Venereol 2020;34(5):e212-e213.

3. Matar S, Oules B, Sohier P, Chosidow O, Beylot-Barry M, Dupin N, et al. Cutaneous manifestations in SARS-CoV-2 infection (COVID-19): a French experience and a systematic review of the literature. J Eur Acad Dermatol Venereol Jun 26;10.1111/jdv.16775. doi: 10.1111/jdv.16775. Online ahead of print. 